\title{
Effective Aggregate Data Collection and Enhanced Network Lifetime Using Energy Efficient Aggregation Data Convening Routing in Wireless Sensor Network
}

\author{
Satheesh Kumar D ( $\sim$ Satheesh765424@yahoo.com ) \\ Hindustan College of Engineering and Technology \\ Saravanasundaram $\mathbf{S}$ \\ Hindustan College of Engineering and Technology \\ Prakash S \\ sri sakthi institute of engineering and technology \\ Selvaperumal S \\ Syed Ammal Engineering College
}

\section{Research Article}

Keywords: wireless, Routing, dispersive, Configuration Message, residual energy

Posted Date: September 24th, 2021

DOI: https://doi.org/10.21203/rs.3.rs-926626/v1

License: (c) (1) This work is licensed under a Creative Commons Attribution 4.0 International License.

Read Full License 


\title{
EFFECTIVE AGGREGATE DATA COLLECTION AND ENHANCED NETWORK LIFETIME USING ENERGY EFFICIENT AGGREGATION DATA CONVENING ROUTING IN WIRELESS SENSOR NETWORK
}

\author{
Satheesh Kumar $\mathrm{D}^{1 *}$, Saravanasundaram $\mathrm{S}^{2}$, Prakash $\mathrm{S}^{3}$, Selvaperumal $\mathrm{S}^{4}$ \\ ${ }^{1 *}$ Assistant Professor, Department of Computer Science and Engineering, \\ Hindusthan College of Engineering and Technology, Coimbatore, India \\ Satheesh765424@yahoo.com \\ ${ }^{2}$ Professor, Department of Electrical and Electronics Engineering, \\ Hindusthan College of Engineering and Technology, Coimbatore, India \\ eeesaravana1234@gmail.com \\ ${ }^{3}$ Professor, Sri Shakthi Institute of Engineering and Technology, Coimbatore, India \\ ps4867749@gmail.com \\ ${ }^{4}$ Professor, Department of Electrical and Electronics Engineering, \\ Syed Ammal Engineering College, Ramanathapuram, India. ammselva555@ rediffmail.com
}

\begin{abstract}
A wireless sensor network is a network system that uses wireless sensor nodes to monitor physical or environmental conditions as voice, temperature, and spatial dispersive movements. Each node can locally sense its environment, process information and data and send the data to one or more collection points within the WSN. In the existing solution categorized into member nodes and group/cluster heads $(\mathrm{CH})$. The $\mathrm{CH}$ election process increases the overhead of the network and reduce the network lifetime. The processing and energy limitations of the nodes are considered for the $\mathrm{CH}$ election process. In this cluster formation methods aiming at Cluster head selection process and providing trust in hierarchical WSN are proposed. In this Energy Efficient Aggregation Data Convening Routing (E2ADCR) to estimate the routing path, and aggregate data collection to improve the network lifetime. The major advantage of this technique is to avoid the malicious or selfish node from becoming a dominant cluster in a group of clusters. Initially sink node selection is forward the Configuration Message (CM) to every node on network to construct
\end{abstract}


the performing node. In this, cluster selection based on connection density, degree of the node angle, and residual energy (Quality Factor) that is evaluated from the link robustness, energy and degree of the node. Multi hop link transmission support path optimization technique is estimated in the path when the obstacle is present in the WSN. To introduce an Aggregated Support based Data Collection for evaluate each packet flow monitor on the network if any unrelated packet that will eliminate to forward to sink node. The new routing protocols, which were developed during this research, have better energy efficiency. The proposed routing path of the computational simplicity is achieved by a simple method.

\section{Introduction}

The development of efficient wireless communication and advances in electronic information technology, wireless sensor networks (WSN) are widely used in various fields because of its low cost, miniaturization and multi-functional characteristics. However, in most cases, the nodes in the wireless sensor network are powered by batteries and are usually deployed in unmanned outdoors or in more dangerous environments, which makes it inconvenient to supplement energy. The cost of redundant deployment and node replacement is also usually higher. Therefore, an effective policy routing is needed to minimize the energy consumption of the network and extend the life of the network.

Traditional strategic routing mainly uses the shortest path from the source node to the transmitted data as quickly as possible, as the energy of the sensor node is primarily used to transmit the received data. Think about how to do it. However, in energy constrained sensor networks, large amounts of data are sent from sink and source nodes in "many-to-one" mode, which can cause serious "funnel effects" and "energy holes". As a result, the energy consumption of the nodes located on the shortest left and right paths or sink nodes is much faster and faster than others obtained in the life of energy imbalances and sub-networks.

Aggregation is a communication technique based on the compressed data architectural set. Data communication and data compression methods are used here as energy storage systems. Therefore, the compression ratio is calculated from the data association and cluster size. The collective entropy of sink and compressed messaging depends on the way the $\mathrm{CH}$ aggregators read and send their message to the tank's representative. High gain calibration data collection, sensor nodes to a cluster of very small, usually divided into clusters, which are out of line. Here, from 
each of the cluster nodes, it is called a preamble that is selected, and $\mathrm{CH}$ performs data aggregation. The sensor collects data on the environment through the sending node and other nodes, either directly or at the joint piece. Clustering of sensor nodes, many sensor applications are scalable, powerful, and have reduced network traffic.

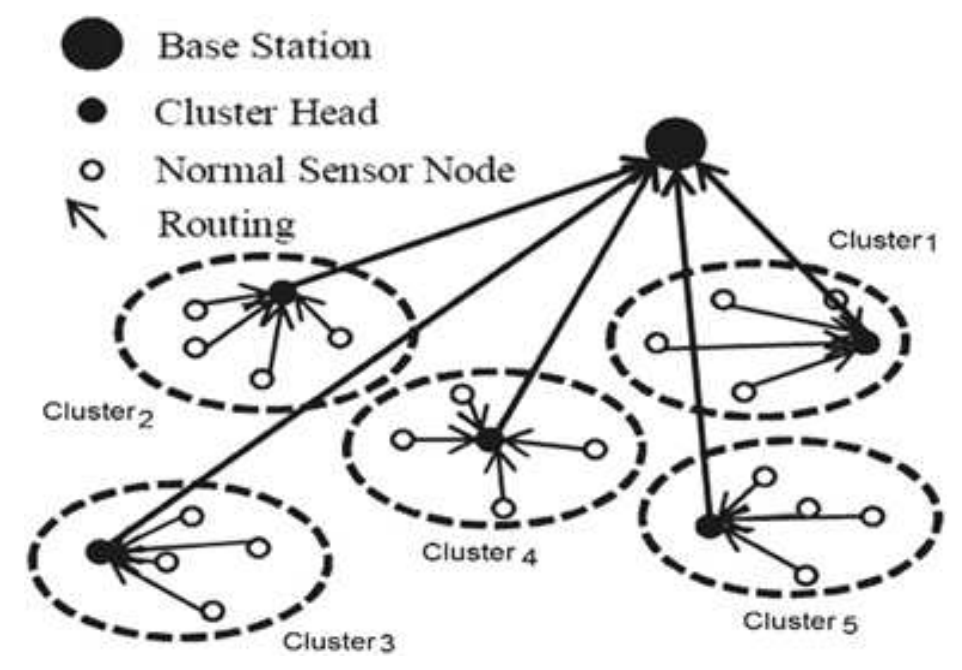

Figure 1 cluster based approach for communication [21]

In WSN, the energy consumed by the sensor is mainly sensed and compared with the processed data that sends and receives the data, and the amount of energy as described in the following data communication is greatly wasted.

The process of dividing a network into interconnect sub-structure is called clusters. Each $\mathrm{CH}$ acts to temporarily connect the other cluster heads with the base station (BS). Each cluster is identified by selected measurements on a specific metric or selective basis (mobility, angle, density). A specific node for each cluster is selected as a metric or a combination of CHs based on the metric identifier. Each $\mathrm{CH}$ acts to temporarily connect the other cluster heads with the BS in that cluster. This information contains a list of nodes within each node of the cluster path. CH's responsibility is to communicate with all nodes in the cluster within the communication network. However, the $\mathrm{CH}$ must be able to communicate with other clusters that can communicate directly or through the corresponding $\mathrm{CH}$ or gateway node. Communication takes place in three stages. Initially, $\mathrm{CH}$ receives the data sent by its members. The compressed data and final data are then 
sent to the base station or other $\mathrm{CH}$. Appropriate $\mathrm{CH}$ can reduce energy use and improve the life cycle of the network.

\section{Related Work}

In [1], a new type of tree routing called event detection tree (EDT) has been proposed in order to achieve savings and high efficiency and energy complex event detection. EDT at the cost of data transmission over increasing distances, reduces the amount of data to be transmitted by aggregating data in the event of achieving this aggregation. Routing protocol that belong to the first category, furthermore, can be divided into flat or hierarchical. Based on the coherent routing protocol, only [2] minimal processing is a highly efficient energy saving mechanism performed by the sensor node. Routing-based processing with non-coherent data, the sensor node processes the actual data locally and sends it to other nodes for further processing. In [3] discussed Bypassing Void Routing Protocol Based on Virtual Coordinate (BVR-VRC) using an edge network topology without routing the gap. BVR-VRC adjusts the mapping to solve the problem of virtual gaps using gap detection, and then establishes a path based on the virtual coordinates of the edge nodes around the gap.

This routing protocol used in wireless sensor networks is being considered more uniformly [4] than the broader polymorphic ones. A detailed study of the latter is expected to meet the requirements of different applications. Link coordinates of this Random projection-Polar coordinate-Chain routing (RPC) method [5]. This method uses polar coordinates to establish a chain structure that forms a path to find a node, and applies random projections to achieve a compressed dataset. The energy-efficient centroid based routing protocol (EECRP) formal cluster of [6] by solving the problem of managing energy in WSN-assisted IoT based on the distance to the center of gravity. An optimization algorithm based on the number of dead nodes and the number of cluster head nodes.

Linear Path develops an improved protocol for so-called straight-line routing (SLR) using two-hop information, for wireless sensor networks constructed without the help of geographic information. Taking that into account has been proposed to measure the energy in the routing process in the routing process due to the lack of reliability of links [7, 8]. It is impractical to route to this optimal energy constrained network as it requires future knowledge. Keeping Paths Straight Provides a natural way to solve the energy cost issues of existing geographic routing protocols. 
Communication between wireless sensor nodes is handled by the routing protocol. The nature of links, low power consumption and limited dependencies makes design energy and performanceefficient routing protocols for wireless sensor networks a daunting task [9]. Dynamic Source Routing (DSR) is a common protocol commonly used in wireless sensor networks, but it does not provide fault tolerance and energy efficiency. In this paper, the modification is a new fault-tolerant routing and energy-saving protocol that the traditional DSR protocol has been proposed [10].

Acting on a Controller this new routing algorithm establishes a queue based on the distance collected from the nodes and calculates the node closest to each node that sends the data. Simulation results show that the new routing algorithm has better performance [11] by extending the life cycle of the network and improving traffic throughout the network. Also, battery power cannot be transferred from one node to another. The study conducted a final analysis of the energyaware routing protocols, their features, benefits, limitations and classification of energy-efficient routing protocols [12].

Clustering is used as a key technology for the energy efficiency balance of resource fear networks loaded in wireless sensor networks. It supports cluster heads in the longevity network. Wireless sensor networks are relying on multiple guidelines clusters [13] to show that there is a considerably large network of life. A wireless sensor network is a wireless network with the latest trends in which human intervention is complicated by the sensor network system is a type of portable electronic device. In wireless sensor networks, in a different environment-sensitive sensor are grouped together to monitor and control the physical properties of the environment. WSN energy-saving routing algorithms are required to perform a given task while maintaining a long network life cycle [14].

Life-Time (LT), in elevation energy consumption and controller networks, wireless sensor networks, suited to improve energy efficiency. Clustering is widely used to reduce power consumption, and LT is a powerful technique to improve network transmission. Based Clustering Novel Rank Based Clustering (NRC) developed ARSH-FATI Head Cluster Selection (ARSHFATI-CHS) power sensor nodes and the traffic between the base, the top LT order to reduce the lifetime of the available functional network-based [15] should improve. It is a hierarchical cluster head of energy-saving wireless sensor networks based on communication protocol plays a key role. In most collection methods, Cluster Head $(\mathrm{CH})$ to send data to the sink node. Wireless sensor 
network technology has become a traditional energy-optimized. Sensor Networks and choose effective leadership Cluster [16] Ridge Method Cluster Head Selection mode (RMCHS) the new synchronous transfer mode.

Cluster Head replication options for increasing network reliability and data network. Wireless sensor network, based on information received from each cluster head is responsible for the collection of data transmits to the base station. Sensors in a wireless sensor network and type2 fuzzy Logic and three factors (energy, distance, density) are used to select the hierarchical set of cluster heads. Layer routing protocols are based on the first communication. Dividing the network protocol smaller clusters, and create a hierarchical structure of nodes [17-18]. Selected some of the cluster heads (CHS) and collected them, then collecting data from the ME (Mobile Environment) cluster heads and collecting data from regular nodes. Approximately deployed sensor networks, the new $\mathrm{CH}$ option is based on the density of nodes. Surrounded by multi-node deployment nodes [19] is likely to be the cluster head.

The algorithm maintains this information preparation method according to the clustering, cluster head collected data from a cluster node, ready to be transmitted to a mobile environment. This way only need $\mathrm{CH}$ to access each cluster node individually for access. The ME [20] is the optimal path determined by the CCP (Critical Control Point) algorithm by connecting all $\mathrm{CH}$ / Collection Points (CP).

\section{Implementation of the proposed method}

The key aspect of the proposed Energy Efficient Aggregation Data Convening Routing (E2ADCR) algorithm is to perform reliable data collection and eliminating outliers and redundant data from network. In this cluster election and routing algorithm are follow the features of the node to be considered. The sensor node ( $\mathrm{SN}$ ) are even to detect the information and collect to transmit high energy node. In this CS are verify the node request and process the correct order data to be sent further unrelated data eliminate form $\mathrm{CH}$. It performs aggregation data reduction by using a locality sensitive hashing function. To select vice-cluster head is primary head in case failure the node perform a $\mathrm{CH}$. Sink disseminates the event of interest and gathers sensed data from the cluster-heads via the relay nodes. This sink node sends cluster head data to the relay node. 


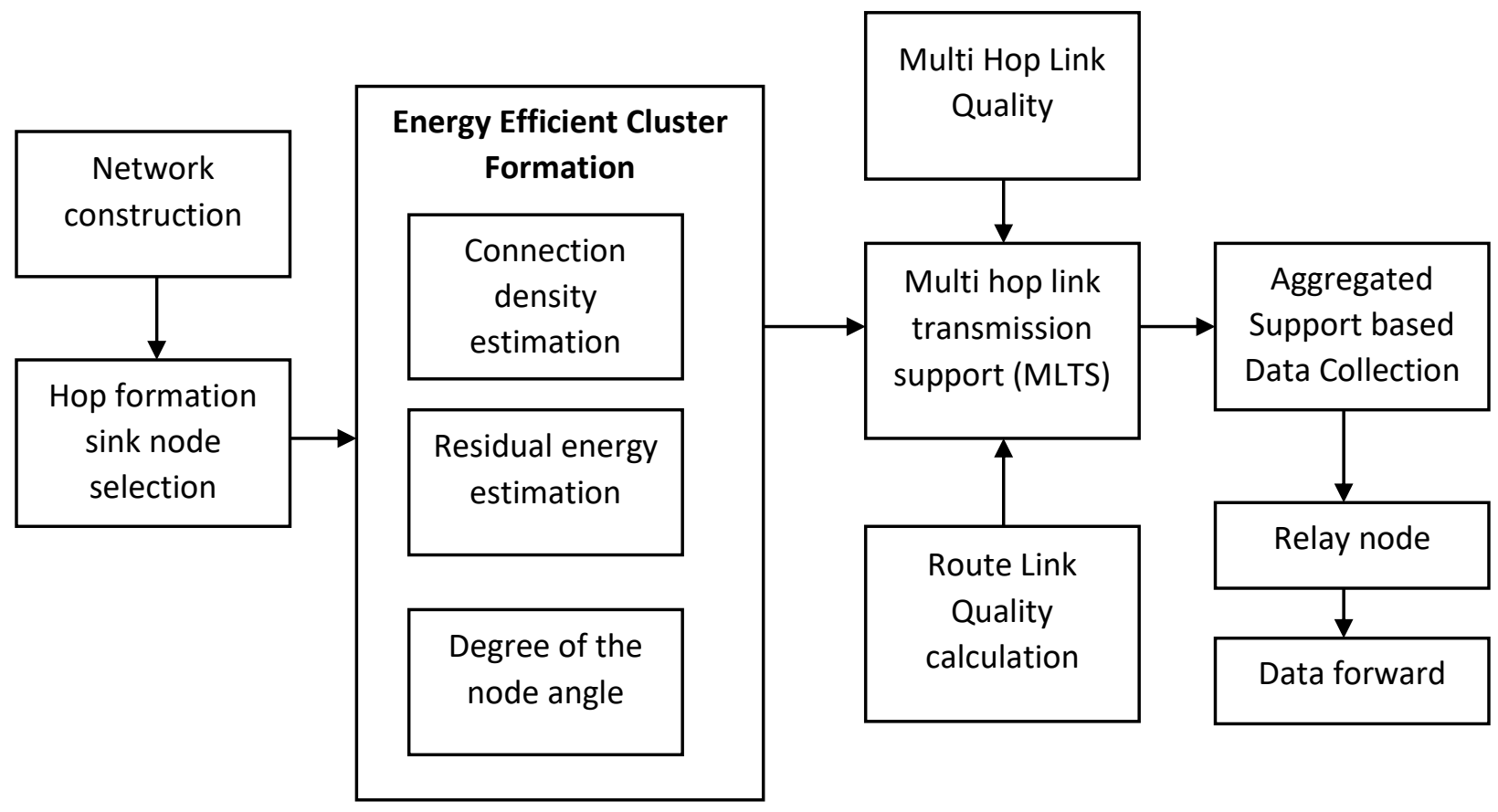

Figure 2 Proposed method Block diagram

In this proposed method to modified selection feature in cluster algorithm to solve the efficient cluster head and reduce the cluster election time. The redundant data and unrelated data eliminate by using aggregation data estimation. In this proposed method performing in three stage there are i) hop formation using sink tree construction, ii) energy efficient cluster formation, iii) Multi hop link transmission support (MLTS) and iv) Aggregated Support based Data Collection. In this process of step process in following section discussed.

\section{a. Hop Formation using sink tree construction}


The sink node establishes a hop for data transfer. The hop distance is calculated from each node shaped sink node to locate the adjacent relay node and cluster head. In this sink tree formation to find the unknown nodes and route discover by using an outbound configuration message to all the node. The configuration message contains two fields: ID and Number of Hops (NH). The configuration message gets the information of node location, identity (ID) and number of hops $(\mathrm{NH})$ are indicate to sink node. In this node information is help to identify the node distance it will be store on database.

\section{Algorithm steps}

Start

Initialize the network node (i)

Broadcast the configuration message $(\mathrm{CH})$ over the network.

Initial hop count $(\mathrm{NH})=1$

The sink node (S) construct the network formation tree based on $\mathrm{CH}$.

If $(i==s)$ then

Compare the $\mathrm{NH}$ value in $\mathrm{CH}$ to configure network $\mathrm{CN}=\mathrm{NH}(i) \in \mathrm{CH}$

$\mathrm{CN}=\mathrm{NH}(\mathrm{i})+1$

If $\mathrm{NH}(\mathrm{I})>\mathrm{NH}(\mathrm{CM})$

$\mathrm{NH}(\mathrm{I}) \leftarrow \mathrm{NH}(\mathrm{CM})+1$

Update the node ID into S, S $\leftarrow$ ID

Node I broadcasts the new CM with updated value to its neighbors

Check the two nodes $\mathrm{i}, \mathrm{j}$

If NH (S) > NH (node I and J)

Check NH $($ node I) $>$ NH $($ node $\mathrm{J})$ 
Discard maximum value of $\mathrm{NH}$

End if

End if

End if

First to initialize the Number of Node $(\mathrm{NH})$ define to infinity and the neighbor node of sink node set as 1 . The configuration information (CM) broadcast help of sink nods to get each node ID and NH value list. All nodes in the network store the minimum NH, which is the minimum distance to the sink.

\section{b. Energy Efficient Cluster Formation}

In Cluster Head $(\mathrm{CH})$ selection phase, all sensor nodes maintain their receivers. Select the cluster head from all the sensor nodes existing in the communication network. The choice of $\mathrm{CH}$ (cluster head) is based on the degree of remaining energy, connection density and node angle. The amount of energy remaining in the node of the current instance is called the remaining energy $\left(E_{\text {res }}\right)$. A node should have more residual energy than its neighbors to become a $\mathrm{CH}$.

Consider $E_{i}$ be the initial energy of the node and $n_{t p}, n_{r p}$ is node transmit and receiving packet. In this node energy consumption $(E(t))$ to be calculated using following equation 1 sequence $\mathrm{T}$ period time.

$$
E(t)=\left(n_{t p} * \alpha\right)+\left(n_{r p} * \beta\right) \quad \text {--- (1) // } \alpha, \beta \text { constant range of the }
$$

node.

Node connection density $\left(c_{n}(\mathrm{x})\right)$ in the same cluster and inter-node distance is calculated at the rate of average distance.

$$
c_{n}(\mathrm{x})=\sum_{i=0}^{n(x, y)}[(x, y) \in E(i) / o(i) \in n(x, y)] \mid n(x, y)
$$

Where, $\mathrm{o}=$ node and $\mathrm{n}(\mathrm{x}, \mathrm{y})$ neighbors.

$$
d(A)=\{d(x)\}
$$


At this initial node angle $\mathrm{d}(\mathrm{x})$ the magnitude of the node position at the cluster node is calculated using Equation 4. In this cluster nodes are located at minimum distance, and higher energy with maximum number of neighbors to have form the network.

\section{c. Multi hop link transmission support (MLTS)}

The multi hop link transmission support is the measure which represent quality of link to perform efficient data transmission. The hops of the route may be moving in different direction and with different node location. To perform efficient data transmission, the hop of the route should be more stable so that the performance can be improved. The stability of the route has been measured in two ways one by multi hop link quality (MHLQ) and Route Link Quality (RLQ) measures. The MLHQ measure represent the quality of link at specific number of hops, because at least for certain number of hops the route should be stable and it would reduce the retransmission frequency.

The list of hops in a route is identified as

$$
\mathrm{Rhl}=\int \text { Hops } \in R
$$

The multi hop constant Mhc is measured as

$$
\operatorname{Mhc}=\frac{3}{4} \times \operatorname{size}(R h l)
$$

The Multi Hop Link Quality

$$
\text { MHLQ }=\frac{\int_{i=1}^{\operatorname{size}(M h c)} \sum M h c(i) \cdot M o b i l i t y<N M T h}{\operatorname{size}(M h c)}
$$

//Nmth-Neighbor Mobility threshold

The Route Link Quality is measured as

$$
\begin{aligned}
\mathrm{RLQ}= & \frac{\sum_{i=1}^{\operatorname{size}(R)} R(i) \cdot \text { Mobility }<T h}{\operatorname{size}(R)} . \\
& / / \text { Here Th-mobility threshold }
\end{aligned}
$$

Finally the MLTS value is measured as

$$
\text { MLTS }=\text { MHLQ } \times \text { RLQ }
$$


Similarly, the RLQ (Route Link Quality) represent the suitability of route in performing efficient transmission. The MLTS measure has been estimated using these two values.

\section{d. Aggregated Support based Data Collection}

The route and node selection to apply the aggregation function all the node and choose the trust data collection in every node. In this method using the elevation of a spatial and temporally relevant database for event-associated data from sensor nodes. Choose the data points used for the end delay and maintain the accuracy of data collection to minimize transmission and eliminate the irrelevant data.

\section{Algorithm steps}

\section{Input: Initialize network node}

\section{Output: selective node (Sn)}

While (node position and node Weight List)

Current Node $\leftarrow$ Node ID- of - Least Weight of the node (WL)

Neighbor list of Current node \{

(N1, N1-distance, N1-energy), (N2, N2- distance, N2-energy).... (Nn, Nn- distance, Nn-energy)

\}

Current Node $-\mathrm{WL}=1$

Temp $=\mathrm{WL}++$

While (temp!=0)

If (Neighbor node ID- Status =is not alive)

Remove the first neighbor from Neighbor list

WLCount $=$ WLCount -1 ;

Else 
Apply aggregate value $\mathrm{Ag}=\sum_{n=0}^{\text {node }} i d\left\{\sin ^{-1}\right.$ trust value $(n)+1 \mid$ node $\left.i d\right\}$

WLCount $=$ WLCount -1

End If

If $(\mathrm{Ag}==$ true $)$

$$
\mathrm{Sn}=\operatorname{limit}_{n \rightarrow \infty}\left(\text { node id }+\frac{\text { aggregate data size }}{\text { total number of data }}\right)^{n}
$$

End if

End While

End While

This reduction information is updated each cluster head and temporal characteristics of events related to the size of the dynamically realized aggregated data.

\section{Result and Findings}

The simulation developed in the NS-2 tool with OTCL (Object Tool Command Language) it's similar to object-oriented language. In these tools used for network QoS parameter analysis like throughput, energy consumption, time delays are evaluated. This section presents a relative analysis of the results obtained therefrom.

Table 1. Proposed method simulation parameters

\begin{tabular}{|l|l|}
\hline Parameters & Values \\
\hline Number of nodes & 300 \\
\hline Data size & $202 \mathrm{mb}$ \\
\hline Packet size & $512 \mathrm{~kb}$ \\
\hline Transmission protocol & $\mathrm{TCP}$ \\
\hline Simulation time & $10 \mathrm{~min}$ \\
\hline Starting energy & 10 joule \\
\hline
\end{tabular}

In this above table 1 is show the proposed method developed simulation parameter to analysis the WSN performance. In this proposed Energy Efficient Aggregation Data Convening Routing (E2ADCR) method result compare to existing methods there are Energy-Efficient Region- 
based Routing Protocol for Low-Power and Lossy routing protocol(ER-RPL), Mode-Switched Grid-Based Sustainable Routing protocol (MSGR) and Energy Efficient Region Source Routing Protocol (ER-SR).

$$
\text { Throughput }=\frac{\text { Packets } \text { Received }(n) * \text { Packet size }}{200}
$$

$\mathrm{n}=$ number of nodes

\section{Table 2 Throughput Performance}

\begin{tabular}{|c|c|c|c|c|}
\hline Time in s & $\begin{array}{c}\text { ER-RPL in } \\
\text { bps }\end{array}$ & MSGR in bps & ER-SR in bps & $\begin{array}{c}\text { E2ADCR in } \\
\text { bps }\end{array}$ \\
\hline 10 & 174 & 192 & 210 & 234 \\
\hline 20 & 246 & 264 & 288 & 306 \\
\hline 30 & 312 & 348 & 384 & 396 \\
\hline 40 & 504 & 516 & 522 & 528 \\
\hline 50 & 546 & 558 & 576 & 588 \\
\hline
\end{tabular}

Table 2 represent a existing method and proposed method throughput ratio. In this analysis of existing methods have lower throughput values than ER-SR because the data is sent over an unrecognized relay gap base station, especially at the border. The throughput of the proposed E2ADCR method is improved by increasing the number of nodes.

$$
\text { Normalized Routing overhead }(N R O)=\frac{\text { number of routing packet }}{\text { number of data packet }} * 100
$$




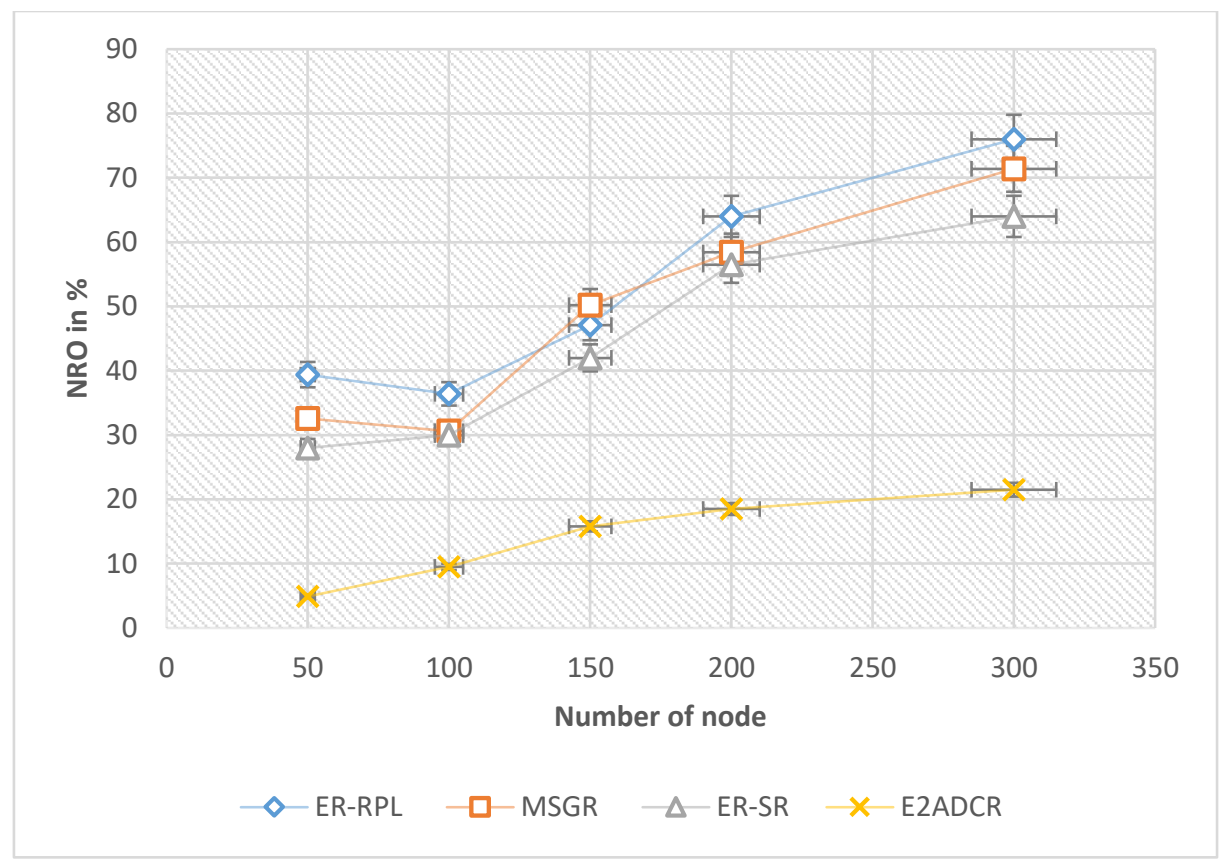

Figure.3 Analysis of normalized Routing Overhead

In this figure 3 is represent a network normalized routing overhead of existing ER-RPL, MSGR, and ER-SR compared to proposed E2ADCR method. In this analysis of proposed E2ADCR method provide a $21.5 \%$ less routing overload for 100 nodes. Similarly, the existing methods ER-RPL, MSGR, and ER-SR have a 76\%, 71.6\%, and 64\% of higher normalized routing overload for 100 nodes.

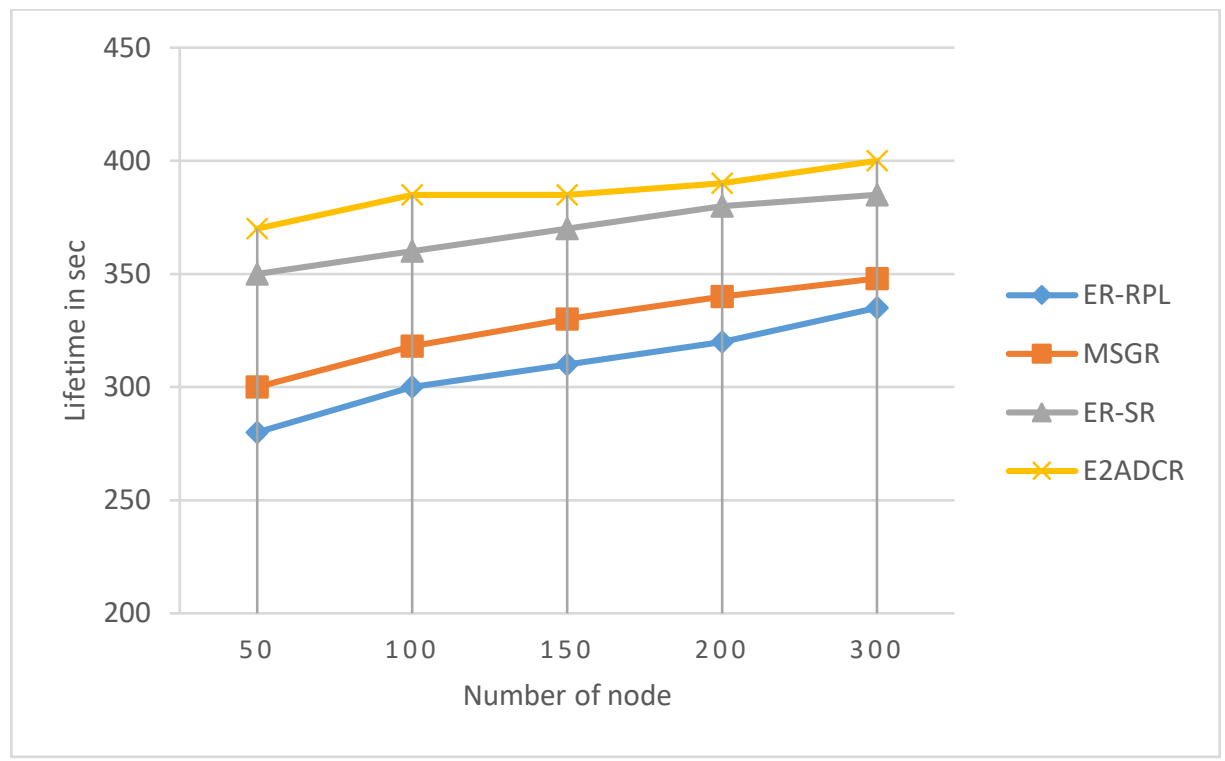

Figure.4 Analysis of network lifetime 
In this analysis, result proposed method has a $400 \mathrm{sec}$ higher network life time compared to the existing method ER-RPL, has $335 \mathrm{sec}$, MSGR has $348 \mathrm{sec}$, and ER-SR has $385 \mathrm{sec}$ of low network lifetime. In this proposed method and existing method ER-RPL, MSGR, ER-SR method result analysis comparison is shown in figure.

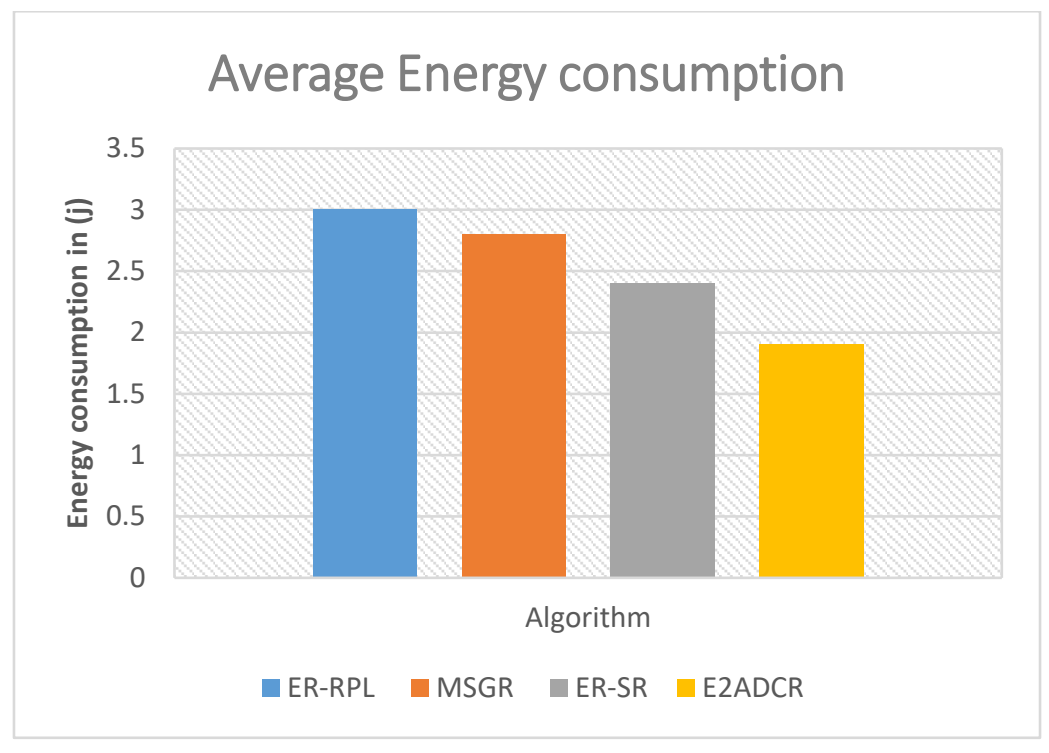

\section{Figure 5. Analysis of Average Energy consumption}

Figure 5 shows the average energy consumption of existing ER-RPL, MSGR, ER-SR and proposed E2ADCR method. In this result, the proposed method has $1.9 \mathrm{j}$ of average energy consumption. Similarly, the existing method ER-RPL, MSGR, ER-SR a 3joule, 2.8joule, and 2.4joule of average energy consumption over the WSN.

\section{Conclusion}

Cluster-based energy efficiency protocol is critical for ensuring $\mathrm{CH}$ is selected. The closest node to choose the ideal position $\mathrm{CH}$ in wireless sensor network after the connection end. The difference between the exact $\mathrm{CH}$ position, and the actual $\mathrm{CH}$ node position, can weaken the energy efficiency capacity. In this proposed Energy Efficient Aggregation Data Convening Routing (E2ADCR) method $\mathrm{CH}$ selection is based on residual energy, connection density, capability of the node and degree of the node. The aggregated Support based Data Collection to help eliminate the irrelevant data form sender to achieve the redundancy data error and higher delivery ratio. In this Multi hop link transmission support (MLTS) method is estimate the link quality and route transmission support to improve the transmission quality in the overall network. This proposed 
method have better result, 588bps of throughput ratio, 1.9joule/s energy consumption with $21.5 \%$ low routing Overhead compare to existing method.

\section{Funding}

There is no funding for this article.

\section{Conflict of interest:}

The authors declare that they have no conflict of interest.

\section{Availability of data and material}

The data and material are taken from energy-efficient centroid based routing protocol using.

\section{Code availability}

The code is a custom code. It was developed by using NS2

\section{Author's contribution:}

The node location and estimate the node is moving position to achieve the network throughput.

\section{Acknowledgements}

The authors would like to thank the editors and anonymous reviewers for their insightful comments and suggestions.

\section{Reference}

1. Zhu, W., Cao, J., \& Raynal, M. (2018). Energy-Efficient Composite Event Detection in Wireless Sensor Networks. IEEE Communications Letters, 22(1), 177-180.

2. Pantazis, N. A., Nikolidakis, S. A., \& Vergados, D. D. (2013). Energy-Efficient Routing Protocols in Wireless Sensor Networks: A Survey. IEEE Communications Surveys \& Tutorials, 15(2), 551-591.

3. Zhang, D., \& Dong, E. (2015). An Efficient Bypassing Void Routing Protocol Based on Virtual Coordinate for WSNs. IEEE Communications Letters, 19(4), 653-656. 
4. Yan, J., Zhou, M., \& Ding, Z. (2016). Recent Advances in Energy-Efficient Routing Protocols for Wireless Sensor Networks: A Review. IEEE Access, 4, 5673-5686.

5. Qiao, J., \& Zhang, X. (2018). Polar Coordinate-Based Energy-Efficient-Chain Routing in Wireless Sensor Networks Using Random Projection. IEEE Access, 6, 21275-21286.

6. Shen, J., Wang, A., Wang, C., Hung, P. C. K., \& Lai, C.-F. (2017). An Efficient CentroidBased Routing Protocol for Energy Management in WSN-Assisted IoT. IEEE Access, 5, 18469-18479.

7. Zhang, X., Tao, L., \& Zhou, F. (2016). Energy Efficient Switch-Based Packet Forwarding for Low Duty-Cycle Wireless Sensor Networks. IEEE Communications Letters, 20(5), 990-993.

8. Ahmed, R. E. (2015). A fault-tolerant, energy-efficient routing protocol for wireless sensor networks. 2015 International Conference on Information and Communication Technology Research (ICTRC). doi:10.1109/ictrc.2015.7156450.

9. Junli, F., Yawen, W., \& Haibin, S. (2017). An improved energy-efficient routing algorithm in software define wireless sensor network. 2017 IEEE International Conference on Signal Processing, Communications and Computing (ICSPCC).

10. Chawda, K., \& Gorana, D. (2015). A survey of energy efficient routing protocol in MANET. 2015 2nd International Conference on Electronics and Communication Systems (ICECS). doi:10.1109/ecs.2015.7125055.

11. More, P. R., \& Sankpal, S. V. (2016). Energy aware routing using energy efficient routing protocol in wireless Ad hoc network. 2016 International Conference on Electrical, Electronics, and Optimization Techniques (ICEEOT). doi:10.1109/iceeot.2016.7754885.

12. LIU, X., ZHANG, J., YU, C., LUO, L., \& SHEN, J. (2019). Multi-domain Awareness Energy-efficient Routing Algorithm for Elastic Optical Networks. 2019 28th Wireless and Optical Communications Conference (WOCC). doi:10.1109/wocc.2019.8770565.

13. Jain, K. L., \& Mohapatra, S. (2019). Energy Efficient Cluster head selection for Wireless Sensor Network: A Simulated Comparison, 2019 IEEE 10th Control and System Graduate Research Colloquium (ICSGRC), doi:10.1109/icsgrc.2019.8837086.

14. Vhatkar, S., Shaikh, S., \& Atique, M. (2017). Performance analysis of equalized and double cluster head selection method in wireless sensor network, 2017 Fourteenth 
International Conference on Wireless and Optical Communications Networks (WOCN), doi:10.1109/wocn.2017.8065854.

15. Ali, H., Tariq, U. U., Hussain, M., Lu, L., Panneerselvam, J., \& Zhai, X. (2020). ARSHFATI a Novel Metaheuristic for Cluster Head Selection in Wireless Sensor Networks, IEEE Systems Journal, 1-12,doi:10.1109/jsyst.2020.2986811.

16. Prasath, K. A., \& Shankar, T. (2015). RMCHS: Ridge method based cluster head selection for energy efficient clustering hierarchy protocol in WSN, 2015 International Conference on Smart Technologies and Management for Computing, Communication, Controls, Energy and Materials (ICSTM), doi:10.1109/icstm.2015.7225391.

17. Joshitha, K. L., \& Gangasri, A. (2017). On an effort to enhance lifetime of a regression based clustered network using candidate selection, 2017 International Conference on Trends in Electronics and Informatics (ICEI), doi:10.1109/icoei.2017.8300816.

18. Garg, N., \& Saxena, S. (2018). Cluster Head Selection Using Genetic Algorithm in Hierarchical Clustered Sensor Network, 2018 Second International Conference on Intelligent Computing and Control Systems (ICICCS), doi:10.1109/iccons.2018.8662914.

19. Usha, M., Sreenithi, S., Sujitha, M., \& Swarnalatha, S. (2017). Node density based clustering to maximize the network lifetime of WSN using multiple mobile elements, 2017 International Conference of Electronics, Communication and Aerospace Technology (ICECA), doi:10.1109/iceca.2017.8212783.

20. Prashanth, J. S., \& Nandury, S. V. (2015). Cluster-based rendezvous points selection for reducing tour length of mobile element in WSN, 2015 IEEE International Advance Computing Conference (IACC), and doi:10.1109/iadcc.2015.7154898.

21. https://onlinelibrary.wiley.com/doi/abs/10.1002/dac.3407 